\title{
Two Species of the Local Group Dwarf Spheroidals
}

\author{
Tsutomu T. Takeuchi ${ }^{1} \&$ Hiroyuki Hirashita ${ }^{1}$ \\ Dept. of Astronomy, Faculty of Science, Kyoto University, Sakyo-ku, \\ Kyoto 606-8502, JAPAN
}

\begin{abstract}
We analyzed 10 dwarf spheroidal galaxies (dSphs) in the Local Group, and found two distinct sequences on the $M_{\mathrm{vir}} / L-M_{\mathrm{vir}}$ plane: $M_{\mathrm{vir}} / L \propto M_{\mathrm{vir}}^{1.6}$ for $M_{\mathrm{vir}}<10^{8} M_{\odot}$ whereas $M_{\mathrm{vir}} / L \simeq$ const. for $M_{\text {vir }}>10^{8} M_{\odot}\left(M_{\text {vir }}\right.$ and $L$ are the virial mass and the total luminosity of a dSph, respectively). We interpret the disconuity as the threshold for the gas in dSphs to be blown away by successive supernovae. We succeeded to give a quantitative explanation of the discontinuity mass of $10^{8} M_{\odot}$ as blow-away condition. We further derived the above relation for the low-mass dSphs, assuming that the initial star formation rate of the $\mathrm{dSphs}$ is proportional to the inverse of the cooling time. The relation of high-mass dSphs is also explained along with the same consideration, with the condition that the gas cannot been blown away.
\end{abstract}

\section{Discovery : $M_{\mathrm{vir}} / L-L$ Relation}

First of all, we present the relation between virial mass $M_{\text {vir }}$ and total $V$ luminosity $L$ of 10 Local Group dSphs in Fig. 1. We divide these galaxies into two groups; the lower-mass group (Group L; Draco, Carina, Ursa Minor, Sextans, Sculptor, and Leo II) and the higher-mass group (Group H; Fornax, NGC 147, NGC 185, and NGC 205) The virial mass of the former group is less than $10^{8} M_{\odot}$, while that of the latter is more than $10^{8} M_{\odot}$. We see that $M_{\text {vir }} / L$ of Group $\mathrm{H}$ is almost constant, though the number of samples is small. As for Group $\mathrm{H}$, the constant $M_{\mathrm{vir}} / L$ may be due to the inefficiency of escape of SN-heated gas (Dekel \& Silk 1986; Mac Low \& Ferrara 1998 (MF98); Ferrara \& Tolstoy 1998 (FT98)), since the potential of dark matter (DM) is deep. This is discussed in the next section. On the other hand, for galaxies belonging to Group L, the gas easily escapes out of them once their gas is heated by SNe (Saito 1979a) and OB-star radiation, because of their shallow gravitational potentials. We discuss this point later in $\S 3$.

\footnotetext{
${ }^{1}$ Research Fellow of the Japan Society for the Promotion of Science

${ }^{2}$ The figure is a revised one omitting the data point of Leo I because its value remains uncertain (Mateo et al. 1998). Our conclusion is not seriously affected by this revision. The quantities of other dSphs are summarized in Hirashita et al. (1998b).
} 
Figure 1. The relation between $M_{\text {vir }}$ and $M_{\text {vir }} / L$ of the Local Group dwarf spheroidal galaxies.

\section{Blow-away Condition for Proto-dwarfs}

As discussed by Saito (1979a), SN-driven winds blow the gas in low-mass protogalaxies away because of their shallow potential wells. Recent numerical simulations of SN-driven wind at initial starburst of low-mass galaxies (MF98; FT98) showed that if their gas mass is more than $10^{7} M_{\odot}$, the gas ejection efficiency is very low. This mass corresponds to $M_{\mathrm{vir}}=10^{8} M_{\odot}$ if the mass fraction of baryon is 0.1 . This value agrees with the separation line of the two groups, $\mathrm{H}$ and $\mathrm{L}$. This fact is physically interpreted as follows. Galaxies with $M_{\mathrm{vir}}>10^{8} M_{\odot}$ form stars whose total mass is proportional to the initial gas mass, which leads to the constant mass-to-light ratio (Dekel \& Silk 1986). On the other hand, galaxies with $M_{\text {vir }}<10^{8} M_{\odot}$ blow the gas away soon after the formation of the first-generation stars: If a little fraction of gas becomes stars, the SNe or UVradiation heating resulting from these stars are enough to blow away the rest of the gas (Saito 1979a; Nath \& Chiba 1995). Thus, the members in the low-mass category tend to have little baryonic matter (i.e., higher $M_{\mathrm{vir}} / L$ ).

\section{Low Mass Dwarf Spheroidals}

The virial mass and total luminosity of the lower-mass group (Group L) in Fig. 1 satisfy the relation,

$$
M_{\text {vir }} / L \propto M_{\text {vir }}^{1.6}
$$

In this section, we will derive this relation by considering the physical processes in the formation epoch of dwarf galaxies. For details, see Hirashita et al. (1998a). 
The presence of DM in dSphs is indicated by observations of velocity dispersions (e.g., Mateo et al. 1993; discussion based on the structure formation is given in Flin 1998). Thus, it is reasonable to consider the gas collapse in DM potential to form proto-dSphs. We assume that the distribution of DM is not affected by baryon, since the mass fraction of baryon is much lower than DM.

We assume that the initial star formation rate (SFR) of a dSph is determined by the cooling time $t_{\text {cool }}\left(t_{\text {cool }} \gtrsim t_{\text {grav }}\right.$, where $t_{\text {grav }}$ is the free-fall time $\left.\simeq 1 / \sqrt{G \rho_{\mathrm{DM}}} \sim 10^{7} \mathrm{yr}\right)$. We, here, note that the free-fall time is determined by the DM mass density $\rho_{\mathrm{DM}}$, while the cooling time is determined by the gas density. The cooling time $t_{\text {cool }}$ is determined by the density and temperature of the gas as

$$
t_{\text {cool }} \propto T / \rho_{\text {gas }} \Lambda(T),
$$

where $\Lambda(T)$ is the cooling function. Since the virial temperature of such a low mass galaxy is much lower than $10^{6} \mathrm{~K}$, the cooling is dominated by $\mathrm{H}$ and He recombination (Rees \& Ostriker 1977): $\Lambda(T) \stackrel{\propto}{\sim} T^{-1 / 2}$ (Peacock \& Heavens 1990). From the above assumption that the SFR is determined by the cooling timescale, we obtain the following expression for the $\operatorname{SFR}(\dot{M})$ :

$$
\dot{M} \propto t_{\text {cool }}^{-1} \propto \rho_{\text {gas }} T^{-3 / 2} .
$$

The temperature in the quasistatic collapse phase is determined by virial temperature (Rees \& Ostriker 1977). Thus, the following expression for the temperature is satisfied:

$$
T \propto M_{\mathrm{vir}} / R,
$$

where $R$ is the typical size of the DM distribution. Using the scaling relation of virial mass and size (Saito 1979b; Nath \& Chiba 1995); $R \propto M_{\mathrm{vir}}^{0.55}$, we obtain from relations (3) and (蛋)

$$
\dot{M} \propto M_{\text {vir }}^{-1.33},
$$

where we assume that the initial mass ratio of gas to DM is constant (i.e., $\rho_{\text {gas }} \propto M_{\text {vir }} / R^{3} \propto M_{\text {vir }}^{-0.65}$.) In normal galaxies, the SF is stopped in the epoch of onset of galactic wind, when the thermal energy produced by SNe becomes equal to the binding energy of the galaxy (Arimoto \& Yoshii 1987). However, in dwarf galaxies belonging to the low-mass group, the heating by UV photons from first-generation OB stars is large enough to supply the thermal energy equal to the gravitational potential ( $\sim 10$ OB stars $\sim 1$ OB association). Thus the remaining gas in a low-mass dwarf evaporates from the system soon after the formation of first-generation stars. We estimate the SF-terminating time by using the crossing time of the wind generated in an $\mathrm{OB}$ association. The velocity of the wind is estimated by the sound speed $c_{\mathrm{S}}$ of $10^{6} \mathrm{~K}$, which is a typical temperature of the heated gas. The crossing time $t_{\text {cross }}$ is estimated as $t_{\text {cross }} \simeq R / c_{\mathrm{s}}$. If we keep $c_{\mathrm{s}}$ constant, the following expression for the $t_{\text {cross }}$ is obtained by using the above scaling law $R \propto M_{\text {vir }}^{0.55}$ :

$$
t_{\text {cross }} \propto M_{\text {vir }}^{0.55} .
$$


We note that the typical value for $t_{\text {cross }}$ becomes $10^{7} \mathrm{yr}$ (for $R=1 \mathrm{kpc}$ and $c_{\mathrm{s}}=100 \mathrm{~km} \mathrm{~s}^{-1}$ ), which is shorter than the cooling time. Thus, the formation of the second-generation stars is difficult. The total mass of the stars, $M_{*}$, is estimated by $M t_{\text {cross }}$. Thus, from relations (5) and (6), we obtain $M_{*} \propto M_{\text {vir }}^{-0.78}$. This relation means that the mass-to-light ratio is scaled as

$$
M_{\mathrm{vir}} / L \propto M_{\mathrm{vir}}^{1.78}
$$

where $L$ is considered to be proportional to $M_{*}$.

Comparing the observed relation (11) with the theoretical prediction (77), we see that the two agree well in the range of the observational error. We note that it is difficult to obtain an absolute value for the mass of the formed stars, since the star formation efficiency is uncertain. Thus, we here only calculate the scaling relation.

Acknowledgments. We are grateful to Drs. A. Ferrara, M.-M. Mac Low and E. Tolstoy for their kindly sending us their preprints and helpful comments on the starburst-driven mass loss. We also thank Drs. K. Freeman, M. G. Lee, and S. van den Bergh for the useful comments on Fig. 1. We acknowledge the Research Fellowship of the Japan Society for the Promotion of Science for Young Scientists.

\section{References}

Arimoto, N. \& Yoshii, Y., 1987, A\&A, 173, 23

Dekel, A. \& Silk, J. 1986, ApJ, 303, 39

Ferrara, A. \& Tolstoy, E. 1998, in preparation (FT98)

Flin, P. 1998, this issue

Hirashita, H., Takeuchi, T. T., \& Tamura, N. 1998a, ApJ, 504, L83

Hirashita, H., Kamaya, H., \& Takeuchi, T. T. 1998b, MNRAS, submitted

Mac Low, M.-M. \& Ferrara, A. 1998, ApJ, submitted astro-ph/9801237) (MF98)

Mateo, M., Olszewski, E. W., Pryor, C., Welch, D. L., \& Fischer, P. 1993, AJ, 105,510

Mateo, M., Olszewski, E. W., Vogt, S. S., \& Keane, M. J. 1998, astro-ph/9807296

Nath, B. B. \& Chiba, M. 1995, ApJ, 454, 604

Peacock, J. A. \& Heavens, A. F. 1990, MNRAS, 243, 133

Rees, M. J. \& Ostriker, J. P. 1977, MNRAS, 179, 541

Saito, M. 1979a, PASJ, 31, 193

Saito, M. 1979b, PASJ, 31, 181 


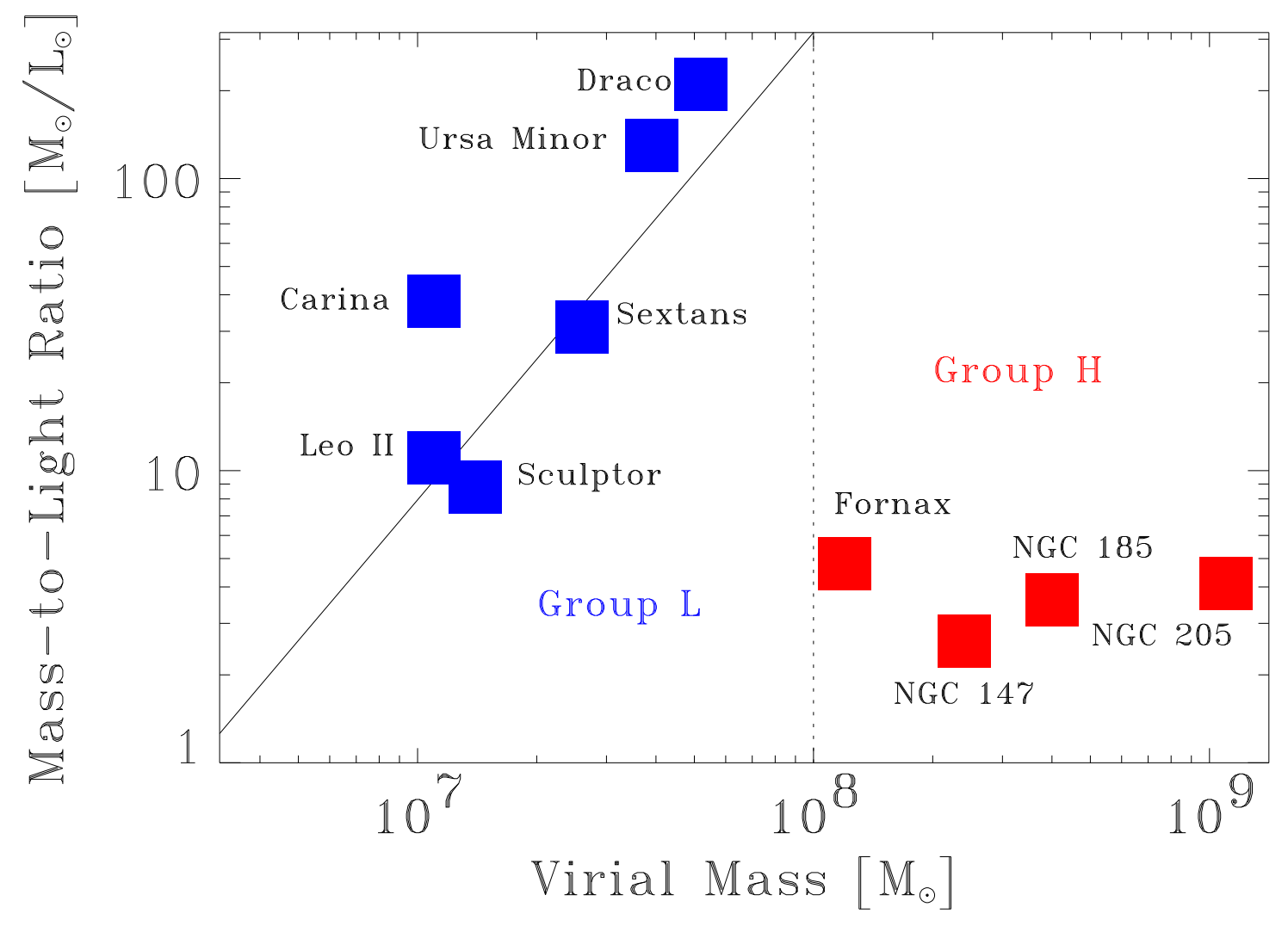

